\title{
CASE REPORT OF TYPE 4 RENAL TUBULAR ACIDOSIS ASSOCIATED WITH ACTIVE SYSTEMIC LUPUS ERYTHEMATOSUS
}

Taíssa Cerqueira dos Santos ${ }^{1, \star}$, Thaynah Pisani Lisboa ${ }^{1}$, Pedro Serra de Candol $^{1}$, Melissa Adriana Torres Gonzalez ${ }^{1}$, Eduardo Florim Terra ${ }^{1}$, Francinne Machado Ribeiro ${ }^{1}$

1.Hospital Universitário Pedro Ernesto, Rio de Janeiro (RJ), Brazil.

*Corresponding author: taissa.cerqueira@gmail.com

\section{BACKGROUND}

Renal tubular acidosis (RTA) is a group of disorders expressed by metabolic acidosis with a normal anion gap that has four distinct types. This condition results from many causes such as drugs and autoimmune diseases. When present in patients with systemic lupus erythematosus (SLE), classic distal RTA (type 1) is the most common. In this population, the occurrence of type 4 hyperkalemic RTA is very rare and represents a diagnostic challenge.

\section{CASE REPORT}

A 38-year-old male diagnosed with SLE in 2011 due to inflammatory polyarthritis, oral ulcers, photosensitivity, lymphadenomegaly, weight loss, ANA positive 1:1280 homogeneous nuclear pattern and hypocomplementemia. Clinical investigation excluded other differential diagnoses such as tuberculosis and malignancy. Besides that, the patient presented two episodes of proliferative glomerulonephritis in 2012 and 2015, diagnosed by histological class inference, when he underwent cyclophosphamide induction therapy and maintenance with mycophenolate mofetil. In May 2020, the aforementioned symptoms resurged in addition to night sweating and dry cough. Complementary tests revealed anemia, leucopenia, lymphopenia, decline of renal function with creatinine clearance of $51.2 \mathrm{~mL} / \mathrm{min} / 1.73 \mathrm{~m}^{2}$ (previous: 115 ), increased proteinuria, hyperkalemia (initial $\mathrm{K}+: 6.8 \mathrm{mEq} / \mathrm{L}$ ), hyperchloremia and severe metabolic acidosis (serum $\mathrm{pH}<7.16$ ) with normal anion gap. Urine sediment examination showed 20,000 red blood cells with $30 \%$ of dysmorphism. The urinary anion gap was positive ( $31.1 \mathrm{mEq} / \mathrm{L}$ ) and urinary $\mathrm{pH}$ was 5.0 . Excluding the most likely alternative causes, including use of medications like angiotensin-converting enzyme inhibitors and cyclosporine, the diagnosis of type IV RTA secondary to SLE was suggested. After initial management of hyperkalemia/acidemia, corticotherapy with methylprednisolone at a dose of $1 \mathrm{mg} / \mathrm{kg} / \mathrm{day}$ was prescribed with patient's improvement in only four days. Moreover, immunosuppression with cyclophosphamide was introduced with complete remission of renal activity after the second dose and the patient was discharged from hospital to follow-up care in an outpatient clinic.

\section{CONCLUSION}

Despite the well-recognized glomerular implications of SLE, lupus nephritis can present itself as RTA as reported in the case above. Although infrequent, the identification of this entity after excluded the most probable causes is imperative since the treatment relies not only in correcting the biochemical disturbances, but rather includes treatment of the cause. 